\title{
Efecto de Caryodendron orinocense sobre la degradación de la materia seca ${ }^{1}$
}

\section{Effect of Caryodendron orinocense on dry matter degradation}

\author{
Pablo Losada-Aguilar ${ }^{2}$, Aurora Cuesta-Peralta ${ }^{3}$, Juan de Jesús Vargas-Martínez $^{4}$
}

\begin{abstract}
Resumen
El objetivo del presente trabajo fue evaluar la degradación in vitro e in situ de la materia seca al incluir torta de cacay (Caryodendron orinocense) en una dieta a base de Brachiaria dictyoneura. El experimento se desarrolló en el laboratorio de nutrición animal adscrito a la Facultad de Ciencias Pecuarias de la Universidad de Ciencias Aplicadas y Ambientales, Colombia, en julio de 2015. Se evaluaron cuatro tratamientos: B. dictyoneura, torta de cacay y dos mezclas de $B$. dictyoneura: torta de cacay (80:20 y 60:40). En los tratamientos se determinó la concentración química y la degradabilidad de la materia seca y de la proteína cruda en condiciones in vitro e in situ a las 48 horas. Las variables evaluadas fueron analizadas a través de un diseño completo al azar con cuatro tratamientos. La inclusión de torta de cacay en la mezcla disminuyó la concentración de carbohidratos asociados a la pared celular e incrementó los nutrientes de mayor digestibilidad; aumentó la degradabilidad de la proteína cruda y de la materia seca in situ e in vitro en 22, 6 y $38 \%$, respectivamente. En conclusión, la inclusión de torta de cacay en la dieta de B. dictyoneura mejoró la degradación de la materia seca en condiciones in vitro e in situ.
\end{abstract}

Palabras claves: alimentación animal, subproducto agroindustrial, suplementación, torta de cacay.

\begin{abstract}
The objective of the present study was to evaluate the in vitro and in situ dry matter degradation by including cacay cake (Caryodendron orinocense) on a diet based on Brachiaria dictyoneura. The experiment was developed in an animal nutrition laboratory on the Animal Sciences Faculty at the Universidad de Ciencias Aplicadas y Ambientales, Bogotá, Colombia, in July 2015. Four treatments were evaluated: B. dictyoneura, cacay cake and two mixtures of $B$. dictyoneura: cacay cake (80:20 and 60:40). The chemical composition and the in vitro and in situ degradability of the dry matter and the crude protein, at 48 hours were determined. The variables were analyzed through a complete randomized design with four treatments. The inclusion of cacay cake decreased the cell wallassociated with carbohydrate concentration and increased the higher digestibility nutrients; the crude protein and dry matter, in situ and in vitro degradability increased 22, 6 and 38\%, respectively. In conclusion, the inclusion of cacay cake on a diet of $B$. dictyoneura improves dry matter degradation in vitro and in situ conditions.
\end{abstract}

Keywords: animal feeding, by-product, supplementation, cacay cake.

1 Recibido: 8 de julio, 2016. Aceptado: 12 de octubre, 2016. Este trabajo hizo parte del trabajo de grado del primer autor para optar por el título de Zotecnista, Universidad de Ciencias Aplicadas (UDCA), Colombia.

2 Universidad de Ciencias Aplicadas (UDCA), Semillero de Investigación en Nutrición Animal, Calle 222 \#53-02. Bogotá, Cundinamarca, Colombia.pa_loag@hotmail.com

3 Universidad de Ciencias Aplicadas (UDCA), Facultad de Ciencias Pecuarias, Calle 222 \#53-02. Bogotá, Cundinamarca, Colombia. acuesta@ udca.edu.co

4 Corporación Colombiana de Investigación Agropecuaria, CORPOICA. Km 14 vía Bogotá-Mosquera. Mosquera, Cundinamarca, Colombia. jvargasm@corpoica.org.co 


\section{Introducción}

En Colombia, la incorporación de gramíneas mejoradas y de leguminosas en las pasturas, alimentos balanceados en la dieta y programas de mejoramiento genético, son algunos ejemplos que han permitido una mayor intensificación de los sistemas de producción bovinos. Sin embargo, los bajos índices productivos y reproductivos, y los altos costos respecto a otros países hacen que la ganadería colombiana sea poco competitiva en una economía globalizada (Holmann et al., 2003; Castro et al., 2008; Carulla y Ortega, 2016). En este sentido, se deben evaluar alternativas de producción que permitan desarrollar sistemas sostenibles en los que se tenga como objetivo primordial la conservación del ambiente, el incremento en la producción animal y el mejoramiento en la calidad de vida de la población rural. Estas alternativas, deben propender por la utilización de recursos propios y promover la conservación de material genético nacional.

Una opción agroindustrial que se ha privilegiado en la economía colombiana es la siembra de cultivos perennes destinados a la obtención de biodisel, como es el caso de la palma de aceite (Van-Wyngaard et al., 2015). Sin embargo, es necesario conocer otros recursos que permitirían obtener materias primas de interés comercial que puedan ser destinadas a la producción animal.

Algunos trabajos sugieren que la inclusión de subproductos agroindustriales es considerada una opción que permite mejorar la calidad de la dieta, aumentar la productividad animal y disminuir los costos de producción (Sánchez et al., 2010; Van-Wyngaard et al., 2015). En este sentido, es necesario evaluar las características químicas y físicas, los niveles de inclusión y la presencia de factores antinutricionales en estas materias primas, con la finalidad de generar recomendaciones adecuadas que permitan aumentar la eficiencia productiva del animal y la rentabilidad de los sistemas de producción bovina.

El mejoramiento en las características composicionales de la dieta al incluir alimentos que presentan una solubilidad mayor, da como resultado el incremento en la disponibilidad de nutrientes que permiten aumentar la degradabilidad del alimento (Vargas et al., 2014) y mejorar la respuesta productiva del animal (Lee et al., 2002). En este sentido el cacay (Caryodendron orinocense Karsten) se perfila con potencial para la región de la Orinoquía colombiana.

El cacay es una especie arbórea nativa de la Orinoquía y la Amazonía (Cárdenas y Ramírez, 2004), y está distribuido en Colombia, Venezuela, Ecuador, Perú y Brasil. Sus frutos presentan un alto contenido de aceite con elevados niveles de ácidos grasos poliinsaturados. Este aceite se extrae por medio mecánico y es utilizado en la industria farmacéutica. Además, como subproducto de la extracción del aceite, se obtiene la torta. Estudios preliminares han mostrado un contenido importante de proteína cruda y extracto etéreo, lo que la convertirían en un recurso con alto potencial para ser incluido en la alimentación animal (Cisneros y Díaz, 2006).

El cacay presenta un elevado contenido de proteína, caracterizada principalmente por la presencia de albúminas $(51 \%)$, prolaminas (23\%) y globulinas (16\%), presentándose como una fracción de alta digestibilidad y posible inclusión en la dieta de niños y ancianos (Padilla et al., 2010). Sin embargo, existen pocos estudios que hayan evaluado la inclusión de torta de cacay en la alimentación de rumiantes. El objetivo del presente trabajo fue evaluar la degradación in vitro e in situ de la materia seca al incluir torta de cacay (Caryodendron orinocense) en una dieta a base de Brachiaria dictyoneura.

\section{Materiales y métodos}

\section{Materiales evaluados y tratamientos experimentales}

El Brachiaria dictyoneura fue cosechado en época de verano (febrero de 2015), simulando las condiciones de pastoreo, en la finca La Carolina, ubicada en el municipio de Cumaral, departamento del Meta. El predio presenta 
una altitud media de $550 \mathrm{~m}$, una precipitación de $2800 \mathrm{~mm}$ anuales, temperatura media de $21^{\circ} \mathrm{C}$ y una ubicación geográfica con latitud $4^{\circ} 11^{\prime} 16,13^{\prime \prime} \mathrm{N}$ y longitud $73^{\circ} 15^{\prime} 11,6^{\prime} \mathrm{O}$. El forraje provino de un sistema de pastoreo rotacional con 32 días de descanso y tres de ocupación, sin fertilización. Los suelos prevalentes en la región son oxisoles caracterizados por tener un bajo contenido de materia orgánica y pH ácido. Por otra parte, la torta de cacay se adquirió comercialmente en la empresa Kahai SAS.

El forraje y la torta de cacay fueron secados a $60{ }^{\circ} \mathrm{C}$, luego molidos a un tamaño de partícula de $1 \mathrm{~mm}$ empleando un molino de cuchillas (Thomas Willey, Modelo 4), y conservados en frascos de vidrio con tapa para su posterior análisis. Los análisis se realizaron en el laboratorio de nutrición animal, adscrito a la Facultad de Ciencias Pecuarias de la Universidad de Ciencias Aplicadas y Ambientales, situado en la ciudad de Bogotá, departamento de Cundinamarca, Colombia, con una altitud de $2600 \mathrm{msnm}$, temperatura promedio anual de $16{ }^{\circ} \mathrm{C}$, humedad relativa del $71 \%$ y precipitación promedio de $630 \mathrm{~mm}$; en el punto geográfico determinado por latitud $4^{\circ} 47^{\prime} 58,14^{\prime \prime} \mathrm{N} \mathrm{y}$ longitud $72^{\circ} 2^{\prime} 57,41^{\prime \prime} \mathrm{O}$, durante el segundo semestre de 2015. Los tratamientos evaluados en condiciones in vitro o in situ consistieron en dos controles, Brachiaria dictyoneura y torta de cacay, y dos mezclas de forraje y torta cacay en proporciones 80:20 y 60:40 (Cuadro 1). Los procedimientos in vitro e in situ fueron replicados en el tiempo.

Cuadro 1. Proporción (\%) de Brachiaria dictyoneura (Bd) y torta de cacay (Caryodendron orinocense) (TC) utilizados en la evaluación de la degradación in vitro e in situ de la materia seca. Bogotá, Colombia. Julio 2015.

Table 1. Proportion (\%) of Brachiaria dictyoneura (Bd) and cacay (Caryodendron orinocense) cake (TC) used in the in vitro e in situ dry matter degradation. Bogotá, Colombia. July 2015.

\begin{tabular}{ccc}
\hline Tratamiento & Bd & TC \\
\hline 1 & 100 & 0 \\
2 & 80 & 20 \\
3 & 60 & 40 \\
4 & 0 & 100 \\
\hline
\end{tabular}

\section{Evaluación in vitro de la degradabilidad de la materia}

En la evaluación in vitro de la degradabilidad de la materia se empleó el método descrito por Tilley y Terry (1963). El contenido ruminal se obtuvo de un bovino fistulado en el rumen, con una dieta basal de kikuyo (Cenchrus clandestinus previamente denominado Pennisetum clandestinum), sal y agua ad libitum. Todos los procedimientos fueron aprobados por el comité de bioética de la Universidad de Ciencias Aplicadas y Ambientales. El fluido se filtró y conservó en termos gaseados con $\mathrm{CO}_{2}$ y precalentados a $37^{\circ} \mathrm{C}$. En tubos de $70 \mathrm{ml}$, provistos con tapones de caucho de butilo, se incubaron $0,5 \mathrm{~g}$ de sustrato (el forraje y la torta sola o en mezcla), se adicionaron $50 \mathrm{ml} \mathrm{de}$ inóculo, en proporción 4:1 de solución buffer $(\mathrm{pH}$ 6,7) y fluido ruminal, en condiciones anaerobias. Las mezclas fueron incubadas por triplicado y se incluyeron tres tubos blanco de referencia sin sustrato, en un baño de maría a $39{ }^{\circ} \mathrm{C}$ y se agitaron cuatro veces al día durante 48 horas. Finalizada la fermentación, se llevaron a refrigeración los tubos por una hora para detener la actividad microbial. Posteriormente, el contenido del tubo fue filtrado en bolsas de nylon con tamaño de $22 \times 11 \mathrm{~cm}$ y poro de $50 \mu \mathrm{m}$, el residuo se secó a $60{ }^{\circ} \mathrm{C}$ por 48 horas y se conservó en condiciones ambientales para posterior análisis. 


\section{Evaluación in situ de la degradabilidad de la materia}

En la evaluación in situ de la degradabilidad de la materia se empleó el procedimiento descrito por Øskorv et al. (1980). Se hizo un pesaje inicial de las bolsas de nylon. Se registró el número de cada bolsa y se adicionaron 3,0 $\mathrm{g}$ del sustrato (el forraje, la torta y las mezclas) en cada una. Se incluyeron seis bolsas por cada hora de muestreo y seis blanco. Posteriormente, se agruparon según los tiempos de evaluación. Se introdujeron las bolsas en un bovino con cánula ruminal, alimentado con pasto kikuyo, sal y agua ad libitum. Los tiempos de evaluación fueron 0, 6, 24 y 48 horas. Una vez que se cumplió el tiempo establecido para cada grupo se retiraron las bolsas del animal y se lavaron cuidadosamente durante cinco minutos con agua de grifo. Las bolsas fueron secadas a $60{ }^{\circ} \mathrm{C}$ por 48 horas y se conservaron en condiciones ambientales para posterior análisis.

\section{Análisis de laboratorio}

En el forraje, la torta de cacay, las mezclas y los residuos de fermentación se determinó la materia seca (MS) (AOAC, 2005) y la proteína cruda (PC) (AOAC, 2005). También se obtuvo la concentración de extracto etéreo (EE) (AOAC, 2005), cenizas (AOAC, 2005), fibra en detergente neutro (FDN) y ácido (FDA) (Van-Soest et al., 1991). Se calculó la digestibilidad de la materia seca según lo descrito por Blümmel et al. (1997). Además, se determinó la concentración de materia orgánica (MO), el contenido celular (CC), la proteína degradable (PCD) y los carbohidratos no estructurales (CNE) de los tratamientos, según lo descrito por Vargas et al. (2014).

\section{Análisis estadístico}

Los datos de digestibilidad in situ de la materia seca y de la proteína cruda se ajustaron al modelo de Gompertz, como se describe a continuación:

$$
\mathrm{Y}=\mathrm{a} * \exp (-\exp (\mathrm{b}-(\mathrm{c} * \mathrm{t})))
$$

Donde $\mathrm{Y}$ es la digestibilidad en un tiempo t, a $>0$ es la degradabilidad máxima, $\mathrm{c}>0$ es la tasa de degradación, b controla la diferencia entre la degradabilidad inicial y la final a un tiempo t.

Los parámetros de degradación de la materia seca in situ fueron estimados a través del procedimiento nlin de SAS (SAS, 2004). Además, se estimó la hora y la degradabilidad al punto de inflexión, y la tasa máxima de degradación.

Las variables de composición química, degradabilidad de la materia seca in vitro y los parámetros de fermentación in situ fueron evaluados a través de un modelo completo al azar con una fuente de variación y con dos repeticiones según lo descrito por Martínez et al. (2011), empleando el procedimiento glm de SAS (SAS, 2004). Además, se comparó la degradabilidad in situ e in vitro de la materia seca a las $48 \mathrm{~h}$ a través de una prueba T. Para la comparación de medias se utilizó la prueba de Tukey.

\section{Resultados y discusión}

La inclusión de torta de cacay incrementó la degradabilidad in vitro e in situ de la materia seca y de la proteína cruda, esto se debe a un aumento en la concentración de PC, contenido celular (CC), materia orgánica (MO) y carbohidratos no estructurales (CNE), y a la disminución en las concentraciones de FDN, FDA y cenizas (Cuadro 2). El incremento en la concentración de nutrientes solubles en la dieta ha sido asociado con una mayor degradabilidad in vitro, producción de gas y de ácidos grasos volátiles, debido a un aumento de la disponibilidad 
Cuadro 2. Composición química y degradabilidad in vitro de la materia seca de Brachiaria dictyoneura, torta de cacay (Caryodendron orinocense) y las mezclas. Bogotá, Colombia. Julio, 2015.

Table 2. Chemical composition and dry matter presented in vitro degradability in Brachiaria dictyoneura, cacay (Caryodendron orinocense) cake and the mixtures. Bogotá, Colombia. July, 2015.

\begin{tabular}{|c|c|c|c|c|c|}
\hline \multirow[t]{2}{*}{ Variable $(\%)^{1}$} & \multicolumn{4}{|c|}{ Relación Brachiaria:torta de cacay } & \multirow[t]{2}{*}{$\mathbf{P}<$} \\
\hline & 100:0 & $80: 20$ & $60: 40$ & $0: 100$ & \\
\hline PC & $9,0^{\mathrm{d}}$ & $15,2^{\mathrm{c}}$ & $20,8^{\mathrm{b}}$ & $39,2^{\mathrm{a}}$ & $* *$ \\
\hline FDN & $67,1^{\text {a }}$ & $57,3^{\mathrm{b}}$ & $46,7^{\circ}$ & $22,6^{\mathrm{d}}$ & $* *$ \\
\hline FDA & $31,5^{\mathrm{a}}$ & $27,1^{\mathrm{b}}$ & $22,5^{\mathrm{c}}$ & $7,6^{\mathrm{d}}$ & $* *$ \\
\hline $\mathrm{EE}$ & $2,0^{\mathrm{d}}$ & $2,9^{\mathrm{c}}$ & $5,1^{\mathrm{b}}$ & $8,1^{\mathrm{a}}$ & $* *$ \\
\hline $\mathrm{CN}$ & $7,9^{\mathrm{a}}$ & $7,4^{\mathrm{ab}}$ & $6,7^{\mathrm{b}}$ & $6,3^{b}$ & $*$ \\
\hline $\mathrm{CC}^{2}$ & $32,9^{\mathrm{d}}$ & $42,7^{\circ}$ & $53,3^{\mathrm{b}}$ & $77,5^{\mathrm{a}}$ & $* *$ \\
\hline $\mathrm{HC}^{2}$ & $35,6^{\mathrm{a}}$ & $30,2^{\mathrm{b}}$ & $24,2^{\mathrm{c}}$ & $14,9^{\mathrm{d}}$ & $* *$ \\
\hline $\mathrm{CNE}^{2}$ & $14,2^{\mathrm{d}}$ & $17,3^{\mathrm{c}}$ & $20,7^{\mathrm{b}}$ & $23,9^{\mathrm{a}}$ & $* *$ \\
\hline $\mathrm{MO}^{2}$ & $92,3^{\mathrm{c}}$ & $92,6^{\mathrm{bc}}$ & $93,3^{\mathrm{ab}}$ & $93,7^{\mathrm{a}}$ & $*$ \\
\hline DIVMS $_{24}$ & $42,2^{\mathrm{d}}$ & $58,5^{\mathrm{c}}$ & $67,7^{\mathrm{b}}$ & $90,4^{\mathrm{a}}$ & $* *$ \\
\hline DIVMS $_{48}$ & $51,0^{\mathrm{d}}$ & $62,1^{\mathrm{c}}$ & $71,6^{\mathrm{b}}$ & $90,8^{\mathrm{a}}$ & $* *$ \\
\hline
\end{tabular}

${ }^{1}$ PC: proteína cruda. FDN: fibra en detergente neutro. CC: contenido celular. FDA: fibra en detergente ácido. HC: hemicelulosa. CN: cenizas. MO: materia orgánica. EE: extracto etéreo. CNE: carbohidratos no estructurales. DIVMS ${ }_{24}$ : degradabilidad in vitro de la materia seca a las 24 horas. DIVMS 48 : degradabilidad in vitro de la materia seca a las 48 horas / PC: crude protein. FDN: neutral detergent fiber. CC: cellular content. FDA: acid detergent fiber. HC: Hemicellulose. CN: ash. MO: organic matter. EE: ether extract. CNE: non-structural carbohydrates. DIVMS ${ }_{24}$ : In vitro dry matter degradation in 24 hours. DIVMS 48 : In vitro dry matter degradation in 48 hours.

${ }^{2}$ CC: 100-FDN. HC: FDN-FDA; CNE: 100-(PC+CN+EE+FDN); MO: 100-CN.

${ }^{a b c}$ Letras diferentes entre columnas, indican diferencias significativas entre tratamientos. $* \mathrm{p}<0,05 . * * \mathrm{p}<0,01 / \mathrm{Different}$ letters between columns, indicate significant differences between treatments. $* \mathrm{p}<0.05$. $* * \mathrm{p}<0.01$.

de nutrientes fermentables para los microorganismos ruminales (Vargas et al., 2013). Esto explicaría la reducción en la degradabilidad de la materia seca in situ e in vitro de la Brachiaria respecto a la mezcla que incluyó 40\% de torta de cacay, en 6,4 y $28,8 \%$, respectivamente (Cuadro 2 y 3 ).

La inclusión de solo torta de cacay aumentó la degradación máxima de la materia seca (A), la tasa de degradación (C), la degradación al punto de inflexión (DPI) y la tasa máxima de degradación (TMD) en comparación a la inclusión de solo Brachiaria en la dieta (Cuadro 3). Como se mencionó anteriormente, el incremento en la concentración de nutrientes de mayor solubilidad y la disminución en la concentración de pared celular (Cuadro 2), promueven la actividad de los microrganismos y es de esperar una mayor degradabilidad del material incubado (Sánchez et al., 2010).

La degradación de la materia seca no debe ser el único indicador que refleje la calidad de la dieta. La dinámica y la sincronía de degradación de los nutrientes permiten una mayor eficiencia en la digestibilidad del alimento, resultando en una mayor disponibilidad de nutrientes para los microorganismos del rumen y el hospedero (Russell et al., 1992). Es por esto que, los modelos de simulación de las dinámicas ruminales consideran las diferentes tasas de degradación de los nutrientes, lo cual permite explicar de una manera más aproximada los procesos de fermentación ruminal (NRC, 2001; Van-Amburgh et al., 2015). En este sentido, la alimentación de rumiantes 
Cuadro 3. Parámetros de degradación in situ de la proteína cruda y la materia seca en Brachiaria dictyoneura, torta de cacay (Caryodendron orinocense) y las mezclas. Bogotá, Colombia. Julio, 2015.

Table 3. In situ degradation parameters of the crude protein and dry matter in Brachiaria dictyoneura, cacay (Caryodendron orinocense) cake and the mixtures. Bogotá, Colombia. July, 2015.

\begin{tabular}{|c|c|c|c|c|c|}
\hline \multirow[t]{3}{*}{ Variable $^{1}$} & \multicolumn{4}{|c|}{ Relación Brachiaria:torta de cacay } & \multirow[t]{3}{*}{$\mathbf{P}<$} \\
\hline & 100:0 & $80: 20$ & $60: 40$ & $0: 100$ & \\
\hline & \multicolumn{4}{|c|}{$\%$} & \\
\hline PCD & $77,2^{\mathrm{d}}$ & $89,0^{\mathrm{c}}$ & $94,2^{\mathrm{b}}$ & $99,9^{\mathrm{a}}$ & $* * *$ \\
\hline PCND & $22,8^{\mathrm{a}}$ & $11,0^{\mathrm{b}}$ & $5,8^{\mathrm{c}}$ & $0,1^{\mathrm{d}}$ & $* * *$ \\
\hline A & $71,8^{b}$ & $72,1^{b}$ & $76,7^{b}$ & $98,5^{\mathrm{a}}$ & $* * *$ \\
\hline $\mathrm{C}$ & $0,05^{\mathrm{b}}$ & $0,08^{\mathrm{b}}$ & $0,1^{\mathrm{b}}$ & $0,3^{\mathrm{a}}$ & $* * *$ \\
\hline DPI & $26,4^{b}$ & $26,5^{b}$ & $28,2^{\mathrm{b}}$ & $36,3^{\mathrm{a}}$ & $* * *$ \\
\hline \multirow[t]{2}{*}{ TMD } & $1,4^{\mathrm{b}}$ & $2,2^{\mathrm{b}}$ & $2,7^{\mathrm{b}}$ & $12,2^{\mathrm{a}}$ & $* * *$ \\
\hline & \multicolumn{5}{|c|}{$\mathrm{h}$} \\
\hline HPI & $1,8^{\mathrm{a}}$ & $-1,1^{\mathrm{b}}$ & $-1,4^{\mathrm{b}}$ & $0,7^{\mathrm{ab}}$ & $* * *$ \\
\hline
\end{tabular}

${ }^{1}$ PCD: proteína cruda degradable. PCND: proteína cruda no degradable. A: degradación máxima de la materia seca. C: tasa de degradación. DPI: degradación al punto de inflexión. TMD: tasa máxima de degradación. HPI: hora al punto de inflexión / PCD: degradable crude protein. PCND: non-degradable crude protein. A: maximum dry matter degradation. $\mathrm{C}$ : degradation rate. DPI: degradation at the turning point. TMD: maximum degradation rate. HPI: hours at turning point. abc Letras diferentes entre columnas, indican diferencia entre tratamientos. $* * * \mathrm{p}<0,001 /$ Different letters between columns, indicate differences between treatments. $* * * \mathrm{p}<0.001$.

debe estar enfocada en la degradación sincrónica de los nutrientes, lo que permite una mayor síntesis de proteína microbial y un mayor rendimiento productivo del animal (Miller et al., 2001).

La inclusión de $40 \%$ torta de cacay respecto a la Brachiaria sola aumentó en $22 \%$ la digestibilidad in situ de la PC (Cuadro 3). La literatura reporta que forrajes con bajas concentraciones de PC limitan el consumo potencial de la materia seca y presentan una alta proporción del nitrógeno ligado a la FDA (Minson, 1990), resultando en una menor disponibilidad de nutrientes para el crecimiento de los microorganismos ruminales y la limitada degradación de la materia seca incubada (Sánchez et al., 2010).

Los modelos permiten describir la realidad de una manera matemática y tienen como objetivo comprender y/o predecir un fenómeno (Thornley y France, 2007). La literatura reporta diferentes modelos que describen las dinámicas de fermentación in vitro e in situ (Rodrigues et al., 2009; Wang et al., 2011). Sin embargo, algunos modelos estiman parámetros que no tienen una explicación biológica y dificultan la interpretación (López, 2005). El modelo de Gompertz ha sido utilizado para parametrizar la dinámica de fermentación, aunque es evidente que este tipo de parámetros deben ser empleados con precaución (Molina et al., 2013).

En este trabajo se encontró que la hora al punto de inflexión es negativa cuando se incluyó 20 y $40 \%$ de torta de cacay en una dieta base de Brachiaria. Esta respuesta puede explicarse posiblemente por la interacción entre los nutrientes solubles del forraje y de la torta de cacay que promovieron una dinámica de fermentación de tipo exponencial más que sigmoidea. El modelo de Gompertz supone una fase inicial de lento crecimiento (fase lag), seguida por una fase exponencial y finaliza con una de estabilización (Molina et al., 2013). Esta fase inicial se caracteriza por la colonización del sustrato por parte de los microorganismos ruminales. Sin embargo, al presentarse la sincronía entre nutrientes altamente degradables, esta fase inicial podría presentar un comportamiento exponencial.

La degradabilidad de la materia seca fue diferente entre las dos técnicas empleadas en este trabajo (Figura 1). 


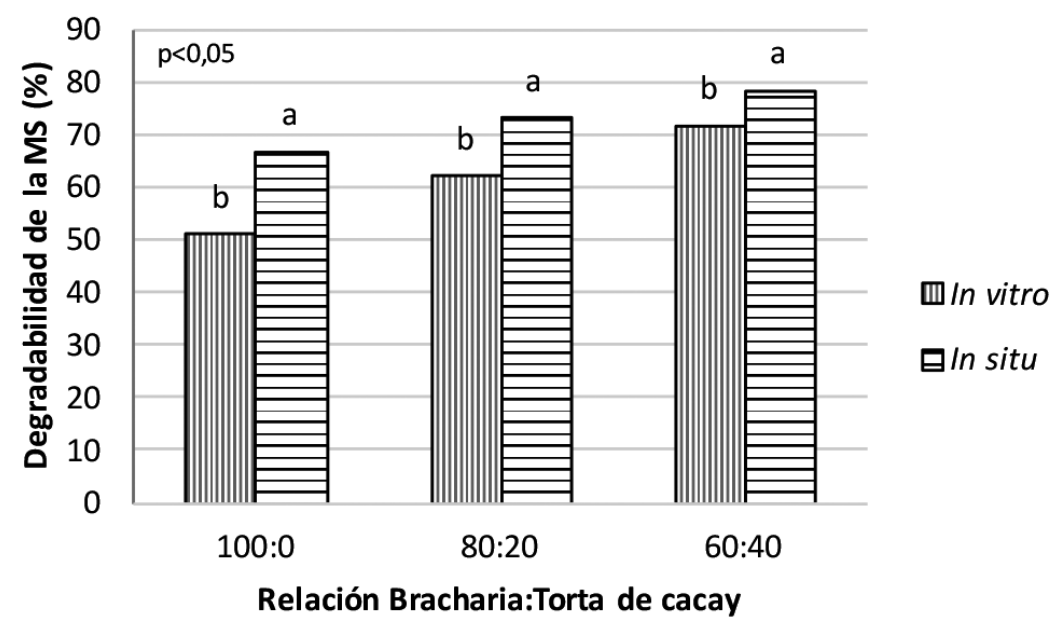

Figura 1. Degradabilidad in vitro e in situ de la materia seca en diferentes proporciones de Brachiaria dictyoneura y torta de cacay (Caryodendron orinocense) a las 48 horas de incubación $(\mathrm{p}<0,05)$. Bogotá, Colombia. Julio, 2015.

Figure 1. In vitro and in situ degradability of the dry matter from different proportions of Brachiaria dictyoneura and cacay (Caryodendron orinocense) cake at 48 hours of incubation (p<0.05). Bogotá, Colombia. July, 2015.

La degradación in situ fue mayor entre 7 y $40 \%$ respecto a la in vitro. Sin embargo, la digestibilidad incrementó al aumentar la inclusión de torta de cacay sin importar la metodología empleada. La técnica in vitro se considera una técnica estática y cerrada, que permite la evaluación de un gran número de muestras en un corto periodo de tiempo (Bhatta et al., 2007), debido a que las condiciones metodológicas de esta técnica pueden variar los procesos de fermentación, como por ejemplo no existe flujo de saliva, absorción de ácidos grasos volátiles o tasas de paso diferenciadas. Esta particularidad resulta en la modificación de poblaciones ruminales (ausencia de protozoarios) y la fermentación ruminal ( $\mathrm{pH}$ alcalinos y altas concentraciones de acetato) respecto a condiciones reales (López, 2005; Posada et al., 2014). Por su parte, la técnica in situ representa la digestibilidad en un solo órgano (en este caso el rumen) desconociendo la fermentación que se realice en otros compartimentos del tracto (colón o ciego) y limitando la posibilidad de evaluar la degradabilidad diferenciada debida al paso del alimento (López, 2005). Además, algunas características de la técnica, como el tamaño de la bolsa y el diámetro del poro, así como el tamaño de molienda del sustrato incubado, influyen en la degradabilidad de los nutrientes (Huntington y Givens, 1995). Es por esto que los resultados de estas metodologías deben ser empleados con precaución, reconociendo las limitaciones de las técnicas, pero sin desconocer el aporte valioso a la explicación de fenómenos que permitan el incremento en la eficiencia y la sostenibilidad de los sistemas de producción animal.

\section{Conclusiones}

En conclusión, la inclusión de torta de cacay mejoró las características composicionales de las mezclas evaluadas. Además, incrementó la digestibilidad de la materia seca y de la proteína cruda tanto en técnicas in vitro como in situ. Sin embargo, estos resultados deben ser evaluados en condiciones in vivo para corroborar el efecto positivo de la inclusión de la torta de cacay en la alimentación de rumiantes. 


\section{Literatura citada}

AOAC (Association of Official Agricultural Chemists). 2005. Official methods of analysis. AOAC, WA, USA.

Bhatta, R., K. Tajima, N. Takusari, K. Higuchi, O. Enishi, and M. Kurihara. 2007. Comparison of in vitro and in vivo techniques for methane production form ruminant diets. Asia-Aust. J. Anim. Sci. 20:1049-1056.

Blümmel, M., H.P. Makkar, and K. Becker. 1997. In vitro gas production: a technique revisited. J. Anim. Physiol. Anim. Nutri. 77:24-34.

Cárdenas, D., y J. Ramírez. 2004. Plantas útiles y su incorporación a los sistemas productivos del departamento de Guaviare (Amazonía colombiana). Caldasia 26:95-110.

Carulla, J.E., y E. Ortega, E. 2016. Sistemas de producción lechera en Colombia: retos y oportunidades. Arch. Latinoam. Prod. Anim. 24(2):83-87.

Castro, E., J.E. Mojica, J.M. León, M.L. Pabón, J.E. Carulla, y E.A. Cárdenas. 2008. Productividad de pasturas y producción de leche bovina bajo pastoreo de gramínea y gramínea + Lotus uliginosus en Mosquera, Colombia. Rev. Med. Vet. Zoot. 55:9-21.

Cisneros, D.E., y A. Díaz. 2006. Obtención de aceite de la nuez de Caryodendron orinocense originaria del departamento del Caquetá en la planta piloto de la Universidad de La Salle. Tesis Lic., Universidad de La Salle, Bogotá, COL.

Holmann, F., L. Rivas, J. Carulla, B. Rivera, L. Giraldo, S. Guzmán, M. Martínez, A. Medina, y A. Farrow. 2003. Evolución de los sistemas de producción de leche y su interrelación con los mercados: un análisis del caso colombiano. Documento de trabajo \#193. CIAT, ILRI, y SLP, Cali, COL.

Huntington, J., and D. Givens. 1995. The in situ technique for studying the rumen degradation feeds: A review of the procedure. Nut. Abst. Review. (Series B) 95:63-93.

Lee, R.F., L.J. Harris, J.M. Moorby, M.O. Humphreys, M.K. Theodorou, J.C. MacRae, and N.D. Scollan. 2002. Rumen metabolism and nitrogen flow to the small intestine in steers offered Lolium perenne containing different levels of watersoluble carbohydrate. Anim. Sci. 74:587-596.

López, S. 2005. In vitro and in situ techniques for estimating digestibility. In: J. Dijkstra et al., editors, Quantitative aspects of ruminant digestion and metabolism. $2^{\text {nd }}$ ed. CABI Publishing, Wallingford, GBR. p. 87-122.

Martínez, R., N. Martínez, y M.V. Martínez. 2011. Diseño de experimentos en ciencias agropecuarias y biológicas con SAS, SPSS, R y Statistix. $1^{\text {a }}$ ed. Fondo Nacional Universitario, Bogotá, COL.

Miller, L.A., J.M. Moorby, D.R. Davies, M.O. Humphreys, N.D. Scollan, J.C. MacRae, and M.K. Theodorou. 2001 . Increased concentration of water-soluble carbohydrate in perennial ryegrass (Lolium perenne L.): milk production from latelactation dairy cows. Grass Forage Sci. 56:383-394.

Minson, D.J. 1990. Forage in ruminant nutrition. $1^{\text {st }}$ ed. Academic Press Inc., CA, USA.

Molina, I.C., J.M. Cantet, S. Montoya, G.A. Correa, and R. Barahona. 2013. In vitro methane production from two tropical grasses alone or in combination with Leucaena leucocephala or Gliricidia sepium. Rev. CES Med. Zootec. 8(2):15-31.

NRC (National Research Council). 2001. Nutrient requirement of dairy cattle. $7^{\text {th }}$ ed. National Academy Press, WA, DC, USA.

Ørskov, E.R., F.D. Hovell, and F. Mould. 1980. The use of nylon bag technique for the evaluation of feedstuffs. Trop. Anim. Prod. 5:195-213.

Padilla, F.C., T. Guédez, M.J. Alfaro, M. Regnault, y A.M. Rincón. 2010. Fraccionamiento y caracterización de las proteínas solubles de la harina de nuez de Barinas (Caryodendron orinocense K.). Rev. Inst. Nac. Hig. 41:38-42. 
Posada, S.L., J.F. Ramírez, y R. Rosero. 2014. Producción de metano y digestibilidad de mezclas kikuyo (Pennisetum clandestinum) - papa (Solanum tuberosum). Agron. Mesoam. 25:141-150.

Rodrigues, A.M., J.W. Cone, L.M.M. Ferreira, M.C. Blok, and C.V.M. Guedes. 2009. Relationship between in situ degradation kinetics and in vitro gas production fermentation using different mathematical models. Anim. Feed Sci. Tech. 151:86-96.

Russell, J.B., J.D. O’Connor, D.G. Fox, P.J. Van-Soest, and C.J. Sniffen. 1992. A net carbohydrate and protein system for evaluating cattle diets: I. Ruminal fermentation. J. Anim. Sci. 70:3551-3561.

Sánchez, T., L. Laméla, y O. López. 2010. Efecto de la suplementación con residuos de destilería del maíz en el comportamiento de novillas en una asociación de gramínea y leucaena. Pastos y Forrajes 33(3). payfo.ihatuey.cu/index.php/pasto/article/ view/604

SAS. 2004. SAS/STAT 9.1.3 User's guide. Version 9.1.3. Version 9.1.3. SAS Institute, Cary, NC, USA.

Tilley, J.M.A., and R.A. Terry. 1963. A two-stage technique for the in vitro digestion of forage crops. J. Brit. Grass. Soc. 18:104111.

Thornley, J.H.M., and J. France. 2007. Mathematical model in agriculture. CABI Publishing, and Cromwell Press, Trowbridge, GBR.

Van-Amburgh, M.E., E.A. Collao-Saenz, R.J. Higgs, D.A. Ross, E.B. Recktenwald, E. Raffrenato, L.E. Chase, T.R. Overton, J.K. Mills, and A. Foskolos. 2015. The Cornell net carbohydrate and protein system: updates to the model and evaluation of version 6.5. J. Dairy Sci. 98:6361-6380.

Van-Soest, P., J. Roberton, and B. Lewis. 1991. Methods for dietary fiber, neutral fiber and no starch polysaccharides in relation to nutrition. J. Dairy Sci. 74:3583-3597.

Van-Wyngaard, J.D.V., R. Meeske, and L.J. Erasmus. 2015. Effect of palm kernel expeller as supplementation on production performance of jersey cows grazing kikuyu-ryegrass pasture. Anim. Feed Sci. Tech. 199:29-40.

Vargas, J.J., E.A. Cárdenas, O.L. Mayorga, M.L. Pabón, and J.E. Carulla. 2013. Changes in methane emissions due species, maturity, and legume proportions in grass:legume mixtures incubated in vitro of Colombian highland forages. In: $\mathrm{C}$. Thomas et al., editors, Advances in animal bioscience: Proceedings of the $5^{\text {th }}$ Greenhouse Gases and Animal Agriculture Conference. Cambridge University Press, Dublin, IRD. p. 512. doi:10.1017/S2040470013000113

Vargas, J., M. Pabón, y J. Carulla. 2014. Producción de metano in vitro en mezcla de gramíneas-leguminosas del trópico alto colombiano. Arch. Zootec. 63:397-407.

Wang, M., S.X. Tang, and Z.L. Tan. 2011. Modeling in vitro gas production kinetics: Derivation of Logistic-Exponential (LE) equations and comparison models. Anim. Feed Sci. Tech. 165:137-150. 